\title{
Role of sialidase in glycoprotein utilization by Tannerella forsythia
}

\section{Correspondence \\ Graham P. Stafford \\ G.Stafford@sheffield.ac.uk}

Received 27 June 2011

Revised 24 August 2011

Accepted 26 August 2011

\author{
Sumita Roy, ${ }^{1}$ Kiyonobu Honma, ${ }^{2}$ C. W. lan Douglas, ${ }^{1}$ Ashu Sharma ${ }^{2}$ \\ and Graham P. Stafford ${ }^{1}$
}

\author{
${ }^{1}$ Oral and Maxillofacial Pathology, School of Clinical Dentistry, Claremont Crescent, \\ University of Sheffield, Sheffield S10 2TA, UK \\ ${ }^{2}$ Department of Oral Biology, School of Dental Medicine, University at Buffalo, \\ The State University of New York, Buffalo, NY 14214, USA
}

\begin{abstract}
The major bacterial pathogens associated with periodontitis include Tannerella forsythia. We previously discovered that sialic acid stimulates biofilm growth of $T$. forsythia, and that sialidase activity is key to utilization of sialoconjugate sugars and is involved in host-pathogen interactions in vitro. The aim of this work was to assess the influence of the $\mathrm{NanH}$ sialidase on initial biofilm adhesion and growth in experiments where the only source of sialic acid was sialoglycoproteins or human oral secretions. After showing that $T$. forsythia can utilize sialoglycoproteins for biofilm growth, we showed that growth and initial adhesion with sialylated mucin and fetuin were inhibited two- to threefold by the sialidase inhibitor oseltamivir. A similar reduction (three- to fourfold) was observed with a nanH mutant compared with the wild-type. Importantly, these data were replicated using clinically relevant serum and saliva samples as substrates. In addition, the ability of the nanH mutant to form biofilms on glycoprotein-coated surfaces could be restored by the addition of purified $\mathrm{NanH}$, which we show is able to cleave sialic acid from the model glycoprotein fetuin and, much less efficiently, 9-O-acetylated bovine submaxillary mucin. These data show for the first time that glycoprotein-associated sialic acid is likely to be a key in vivo nutrient source for $T$. forsythia when growing in a biofilm, and suggest that sialidase inhibitors might be useful adjuncts in periodontal therapy.
\end{abstract}

\section{INTRODUCTION}

The oral cavity houses a complex microbial community capable of progressive colonization of tooth surfaces, which can lead to the major dental diseases of caries and periodontitis (Socransky et al., 1998). Periodontitis is a major cause of tooth loss, affecting over 300 million people worldwide (El-Kheshen, 2009), and bacteria associated with periodontitis are also linked with systemic problems such as endocarditis, atherosclerosis and predisposition to pre-term low birth weight (Tanner \& Izard, 2006). The major bacterial pathogens associated with aggressive periodontitis are known as the 'red complex', which comprises Tannerella forsythia, Porphyromonas gingivalis and Treponema denticola (Socransky et al., 1998). While considerable attention has been paid to the other members of this group, less is known of the virulence mechanisms or physiology of $T$. forsythia.

Our recent work has highlighted a major role for the host sugar sialic acid in the biofilm physiology and host-pathogen

Abbreviations: HRP, horseradish peroxidase; NAM, N-acetylmuraminic acid; SNA lectin, Sambucus nigra lectin. interactions of this key periodontal pathogen (Roy et al., 2010). Sialic acids are commonly present as terminal sugars on the surface of a wide variety of host glycoproteins and eukaryotic cells (Angata \& Varki, 2002; Varki, 1997). The sialic acid residues modulate a variety of biological functions, which include mediating cell-cell interactions, stabilizing glycoprotein structure and masking ligand-binding receptors (Angata \& Varki, 2002; Varki, 1997; Bradshaw et al., 1998). In addition to the identification of a novel transport system (NanOUT), we have highlighted the role of a secreted sialidase enzyme in acquisition of sialic acid from the sialoconjugate sugar sialyl-lactose, which contains sialic acid linked to galactose via an $\alpha-2,3$ or $\alpha-2,6$ linkage, and furthermore to glucose via a $\beta-1,4$ linkage (Roy et al., 2010). Sialidases are a family of glycohydrolases which cleave the terminal sialic acid residue from sialoglycoproteins, and have been shown to play a role in virulence for a range of pathogens, including Streptococcus pneumoniae and Pseudomonas aeruginosa (Corfield, 1992; Reinholdt et al., 1990). Their actions include inducing chemokine release from epithelial cells (Kuroiwa et al., 2009), unmasking sialic acid-masked epitopes for adhesion (Corfield, 1992; Tong et al., 2000), promoting biofilm formation (Soong et al., 2006), and 
degrading host glycoproteins to obtain nutrients (Bradshaw et al., 1994; Byers et al., 1999; King et al., 2006; Severi et al., 2007). We have also shown that the T. forsythia $\mathrm{NanH}$ sialidase is important in interactions with human gingival epithelial cells (Honma et al., 2011) in a mechanism that may expose underlying glycosyl epitopes for adhesion and invasion. However, while interaction with host cells is clearly an important process in periodontal pathogenesis, the ability of periodontal bacteria to form persistent and viable biofilms in vivo is equally pertinent.

It is well documented that the sialidase enzymes of some pathogenic bacteria contribute to virulence, especially of those that reside on and/or invade mucosal surfaces. This is likely due to the abundance of sialic acid on the host glycoproteins in these tissues (Corfield, 1992). Human salivary glycoproteins are no exception, and contain various complex sugar substrates such as mucin and fetuin (Pigman \& Gottschalk, 1966). Mucin contains sialic acid linked via its terminal sugar by a $2-6^{\prime}$ glycosidic bond to $N$ acetylglucosamine (Mizan et al., 2000), whereas fetuin contains both $2-3^{\prime}$ and $2-6^{\prime}$ sialic acid linkages and is more abundant in serum, but is also found in saliva and gingival crevicular fluid (Cointe et al., 1998). We hypothesized that T. forsythia, like other human-dwelling bacteria, might be able to use the sugar substrates available in the oral cavity to obtain food and energy during the formation of dental biofilm.

We therefore set out in this study to examine the role of the $\mathrm{NanH}$ sialidase in biofilm formation using a range of relevant model sialoprotein-coated surfaces, and thus to begin to examine the putative in vivo substrates of this enzyme.

\section{METHODS}

T. forsythia. T. forsythia (ATCC 43037) was routinely grown either in liquid culture [TSB-NAM: $10 \%$ trypticase soya broth (TSB; Oxoid)

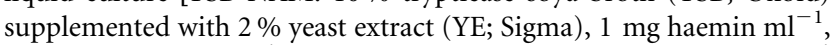
$1 \mathrm{mg}$ menadione $\mathrm{ml}^{-1}$ (Sigma), $10 \mu \mathrm{g} \mathrm{N}$-acetylmuraminic acid $\mathrm{ml}^{-1}$ (NAM; Sigma) and $50 \mu$ g gentamicin $\mathrm{ml}^{-1}$ (Sigma)] or on Fastidious Anaerobe agar plates (FA; Lab M) supplemented with $5 \%$ horse blood containing $10 \mu \mathrm{g}$ NAM ml ${ }^{-1}$ and $50 \mu \mathrm{g}$ gentamicin $\mathrm{ml}^{-1}$. Broth cultures and FA-NAM plates were typically incubated at $37{ }^{\circ} \mathrm{C}$ in an anaerobic atmosphere $\left(10 \% \mathrm{CO}_{2}, 10 \% \mathrm{H}_{2}\right.$ and $\left.80 \% \mathrm{~N}_{2}\right)$ for 5 days. The sialidase mutant $(\Delta n a n H)$ strain was grown in a similar manner but with $5 \mu \mathrm{g}$ erythromycin $\mathrm{ml}^{-1}$ (Honma et al., 2011).

Saliva collection. Resting whole human saliva was collected from healthy individuals aged 25-35 years, centrifuged at $10000 \mathrm{~g}$ for $5 \mathrm{~min}$ and filter-sterilized. The supernatant was used immediately for experiments.

Biofilm growth. T. forsythia biofilms were grown essentially as described previously (Roy et al., 2010). Briefly, 5 day-old T. forsythia colonies were harvested and washed twice in fresh TSB. For biofilm growth, bacteria were inoculated to a final $\mathrm{OD}_{600}$ of 0.05 into the supplemented TSB liquid medium in uncoated polystyrene tissueculture plates (Greiner) and incubated anaerobically at $37{ }^{\circ} \mathrm{C}$ for 5 days. These biofilms were grown (without NAM) either with sialic acid $(6 \mathrm{mM})$ or in the presence of the commercially available glycoproteins bovine submaxillary gland (type I-S), mucin $(6 \mathrm{mM}$, contains $3.8 \mu \mathrm{g}$ sialic acid, molecular mass $484 \mathrm{kDa})$, fetuin $(6 \mathrm{mM}$, contains $0.12 \mu \mathrm{g}$ sialic acid, molecular mass $68 \mathrm{kDa}$ ) and asialofetuin (6 mM, molecular mass $62 \mathrm{kDa}$ ) (all Sigma Aldrich). In addition, human serum (Sigma Aldrich) or fresh whole saliva was used at a dilution of $1: 50$ in PBS $\left(\sim 2 \mu \mathrm{g} \mathrm{ml}^{-1}\right)$ in place of NAM or sialic acid. The glycoproteins were coated on the 96-well tissue-culture plates overnight at $4{ }^{\circ} \mathrm{C}$, and then the wells were washed with PBS to wash off excess protein.

The cell number in mature biofilms was assessed as described previously (Roy et al., 2010); briefly, after 5 days of growth and removal of the culture medium, samples were washed twice in sterile PBS, followed by vigorous resuspension of biofilm cells in PBS using a pipette, before counting microscopically using a Helber counting chamber (Hawksley) under a phase-contrast lens (magnification $\times 400$ ). The effectiveness of the harvesting method was verified by crystal violet staining of the harvested wells to assess the number of residual cells attached to the plate, which was usually $<0.01 \%$ (Pham et al., 2010).

Initial attachment assay. For measurement of T. forsythia adhesion, essentially the same conditions as for mature biofilm growth were used, except that the assays were incubated for only $3 \mathrm{~h}$ at $37{ }^{\circ} \mathrm{C}$ anaerobically. After incubation, the cells were harvested and counted as described above.

Effect of sialidase (neuraminidase) inhibitor on biofilm growth. The effect of the influenza virus sialidase inhibitor oseltamivir on $T$. forsythia biofilm growth was assessed by its inclusion at $10 \mathrm{mM}$ in the medium, followed by the enumeration of biofilm cells after 5 days. This concentration of inhibitor was previously established in our laboratory as suppressing $T$. forsythia whole-cell sialidase activity (Roy et al., 2010). As an alternative, siastatin B was used at a concentration of $5 \mathrm{mM}$ (which inhibits at equivalent levels to the previously utilized oseltamivir), since during the course of this study supplies of oseltamivir were unavailable due to the influenza pandemic.

Purification of recombinant pGEX-NanH. pGEX-nanH (this study) was transformed into Escherichia coli BL21 and a clone was induced using $0.1 \mathrm{mM}$ IPTG. After addition of IPTG, the culture was incubated for $3 \mathrm{~h}$ to express the fusion protein. The cells were harvested by centrifugation, suspended in $200 \mathrm{mM}$ phosphate buffer $(\mathrm{pH} 7)$, and subjected to cell disruption by French pressure cell (1000 p.s.i.; 6.9 MPa). The cell lysates were centrifuged at $10000 \mathrm{~g}$ for $10 \mathrm{~min}$ at $4{ }^{\circ} \mathrm{C}$ to separate the soluble fraction from the cell debris. The recombinant fusion protein $(\mathrm{rNanH})$ was purified from E. coli lysates as described previously (Honma et al., 2011). The purified enzyme was checked for its activity by sialidase assay, with a mean batch activity of approximately 10-20 U.

Sialic acid release assay. Glycoproteins $(6 \mathrm{mM})$ were incubated with $0.78 \mathrm{mg} \mathrm{rNanH} \mathrm{ml} \mathrm{m}^{-1}$ for $3 \mathrm{~h}$ at $37^{\circ} \mathrm{C}$ in $50 \mathrm{mM}$ sodium citrate buffer, $\mathrm{pH}$ 4.5. Free sialic acid was then measured using the method of Skoza \& Mohos (1976). To ensure specificity the sialidase inhibitor siastatin B was included as indicated above.

Direct removal of lectin-binding domains on fetuin. Fetuin $\left(10 \mu \mathrm{g} \mathrm{ml}^{-1}\right)$ was incubated with various concentrations of purified rNanH protein $\left(0,0.78,1.56\right.$ and $\left.3.12 \mathrm{mg} \mathrm{ml}^{-1}\right)$ for $3 \mathrm{~h}$ at $37^{\circ} \mathrm{C}$ in a total volume of $30 \mu \mathrm{l} 50 \mathrm{mM}$ sodium citrate, $\mathrm{pH} 4.5$. The samples were then separated by $10 \%$ SDS-PAGE, transferred to nitrocellulose membranes and incubated with a 1:1000 dilution of biotinylated Sambucus nigra (SNA) lectin (Vector Laboratories), followed by washing in PBS before addition of a 1:10000 dilution of a horseradish peroxidase (HRP)-streptavidin conjugate (Sigma). The SuperSignal West Pico chemiluminescent ECL substrate (Thermo 
Scientific) allowed capture of signal on CL-XPosure film (Thermo Scientific) using a Tetenal developer and fixer in a Compact X4 Xograph imaging system.

Statistical analysis. Comparisons of strains for biofilm growth and adhesion assays, and also the effects of enzyme inhibitors on biofilm growth and adhesion assays, were analysed by Student's $t$ test.

\section{RESULTS}

\section{NanH-dependent sialidase activity is important for biofilm growth using human glycoproteins as a source of sialic acid}

To test whether T. forsythia can obtain sialic acid from relevant host glycoproteins for biofilm growth, 96-well plates were pre-coated with model glycoproteins at a similar concentration to that required for growth on free sialic acid and sialyl-lactose (6 mM) (Roy et al., 2010). In parallel, filter-sterilized human whole saliva from healthy volunteers and human serum (purchased from Sigma) were used to mimic gingival crevicular fluid, as these represent typical secretions present in the oral cavity. Since T. forsythia sialidase activity can be inhibited by oseltamivir (Roy et al., 2010), we tested the effect of this inhibitor on biofilm formation to examine whether any or all of the growth was reliant on sialic acid.

Our previous data showed that biofilm growth on sialyllactose was inhibited fivefold by oseltamivir. We now highlighted the role of the $\mathrm{NanH}$ sialidase in this process, since the nanH deletion mutant was also unable to utilize sialyl-lactose for biofilm growth (Fig. 1). Similarly, biofilm growth on the heavily sialylated bovine submaxillary mucin was also reduced twofold by oseltamivir $(P<0.0001)$ and the nanH deletion $(P<0.005)$ (Fig. 1). While growth on fetuin was only marginally reduced by oseltamivir, the loss of $\mathrm{NanH}$ (in the $\Delta$ nanH mutant strain) caused a striking fourfold reduction $(P<0.0005)$. In support of this observation, growth on asialofetuin compared with fetuin was much lower $(P<0.02)$. In addition, biofilm growth on asialofetuin was higher $(P<0.03)$ than the background when the cells were inoculated into media lacking NAM or sialic acid as a growth substrate, suggesting that the bacteria might use other sugar substrates present on asialofetuin. In order to test whether the sialidase activity had any influence on growth in a situation where there was a clinically relevant mixture of glycoproteins, we repeated the experiment on surfaces coated with human saliva and serum. Under these experimental conditions there were statistically significant two- to threefold reductions (in the presence of inhibitors) in growth
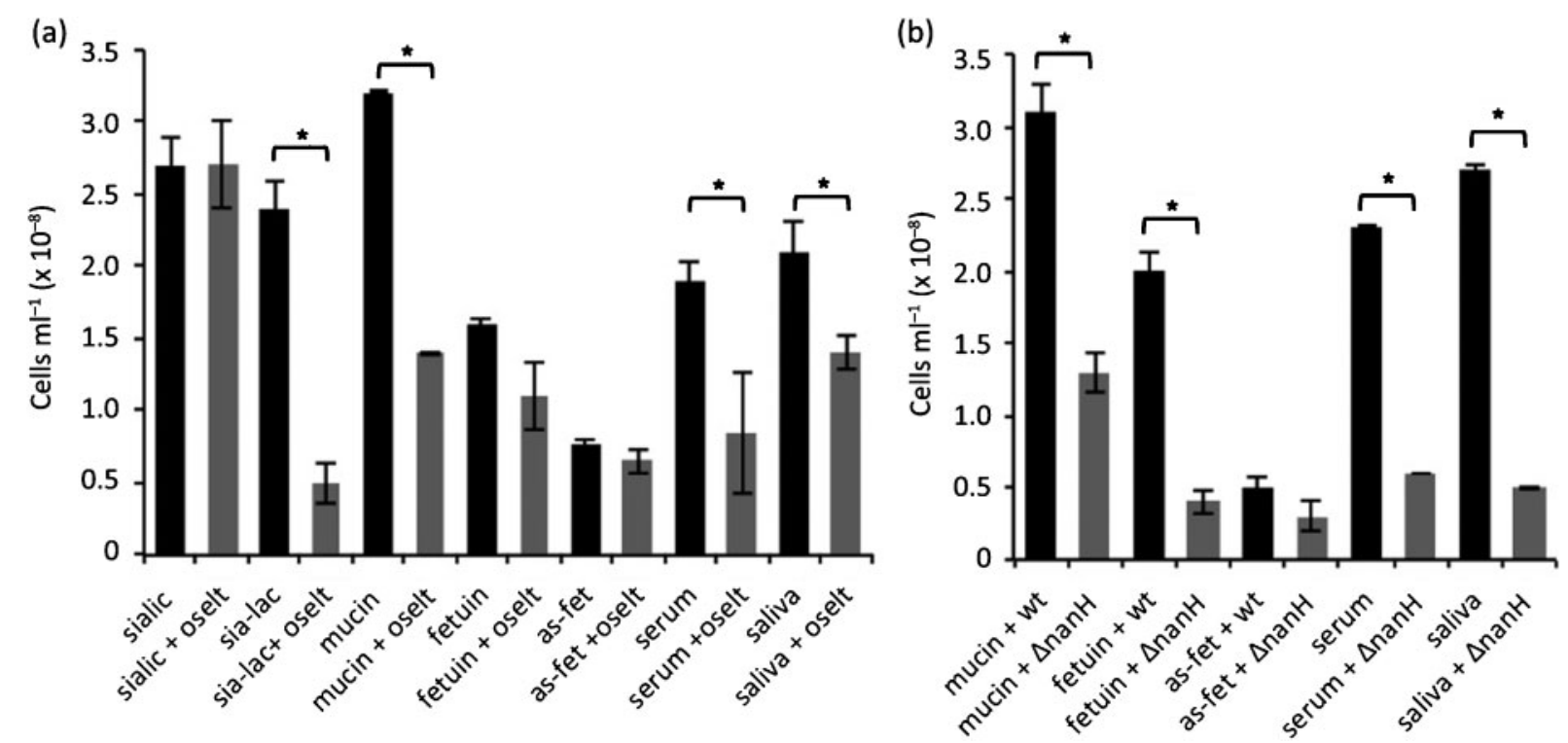

Fig. 1. Growth of $T$. forsythia biofilm on glycoprotein surfaces. (a) Biofilm growth was assessed for the wild-type strain in the absence (black bars) or presence of oseltamivir (grey bars). Biofilms were set up in triplicate and wells were supplemented with $6 \mathrm{mM}$ sialic acid (sialic) or $6 \mathrm{mM}$ sialyl-lactose (sia-lac), or coated with $6 \mathrm{mM}$ mucin, fetuin and asialofetuin (as-fet), $2 \mu \mathrm{g}$ human serum $\mathrm{ml}^{-1}$ (serum) or saliva, with and without the addition of $10 \mathrm{mM}$ oseltamivir to the TSB medium, as indicated at the time of inoculation. Glycoproteins were coated on the 96 -well plate overnight at $4{ }^{\circ} \mathrm{C}$ and washed before inoculation with $T$. forsythia to a final $\mathrm{OD}_{600}$ of 0.05 . Data are the mean number of cells with SDs after harvesting from wells after 5 days. Differences between mean values in this experiment were evaluated by $t$ test, with $P<0.05$ being taken as the level of significance ('statistically significant). (b) Biofilm growth was compared as above between the wild-type (wt) strain and the $\Delta$ nan $H$ mutant strain of $T$. forsythia $(\Delta \mathrm{nanH})$. 
$(P<0.01)$ on human saliva and serum, suggesting that sialic acid might be an important in vivo growth substrate.

\section{NanH is required for efficient adhesion to sialoglycoproteins, saliva and serum}

As is the case for many biofilm-forming bacteria, the initial adhesion to a surface is a key first step in the establishment of a stable community, such as the dental plaque (Kolenbrander \& Palmer, 2004; Scannapieco, 1994). To test adhesion to sialoglycoproteins, saliva and serum bacteria were incubated with coated surfaces as for the biofilm growth experiments, but for just $3 \mathrm{~h}$.

The inclusion of $10 \mathrm{mM}$ oseltamivir in the medium resulted in reduced adherence of $T$. forsythia to the glycoproteins of between two- and fivefold $(P<0.05)$ (Fig. 2a). demonstrating that the sialidase activity of $T$. forsythia is responsible for initial attachment to model sialylated glycoproteins (mucin and fetuin) and to the clinically relevant protein mixtures (serum and whole saliva). Again, adhesion to asialofetuin was lower than to the sialylated form, and no significant reduction was observed in the presence of the inhibitor. It is also worth noting that the variation in absolute levels of adhesion to different substrates may reflect differences in sialic acid content, but could also reflect the concentration of adhered protein, which is very difficult to estimate; nonetheless, the consistent reductions in adhesion in the presence of inhibitor are striking and indicate a role for sialidase in this event. When the adhesion assays were performed with the nanH sialidase mutant, it displayed a similar two- to fivefold reduction in attachment to the glycoproteins compared with the wild-type strain (Fig. 2b).

Ideally, a genetic complementation experiment would be carried out in which the wild-type nanH gene was reintroduced into the nanH mutant strain. However, to date, no one has yet developed a genetic complementation system for T. forsythia. Therefore we used purified $\mathrm{rNanH}$ protein in an enzyme complementation study (Honma et al., 2011). In these experiments the nanH deletion was incubated with glycoprotein-coated surfaces and cell attachment was assayed. Fig. 3 shows a statistically significant increase $(P<0.05)$ in adhesion of between 1.5and twofold in the presence of $\mathrm{rNanH}$. This confirms that $\mathrm{NanH}$ is essential for initial attachment to the glycoproteins present in host cells or in the oral environment.

\section{NanH can directly release sialic acid from glycoproteins}

In order to more fully characterize the role of $\mathrm{NanH}$ in release of sialic acid from a range of glycoproteins, we
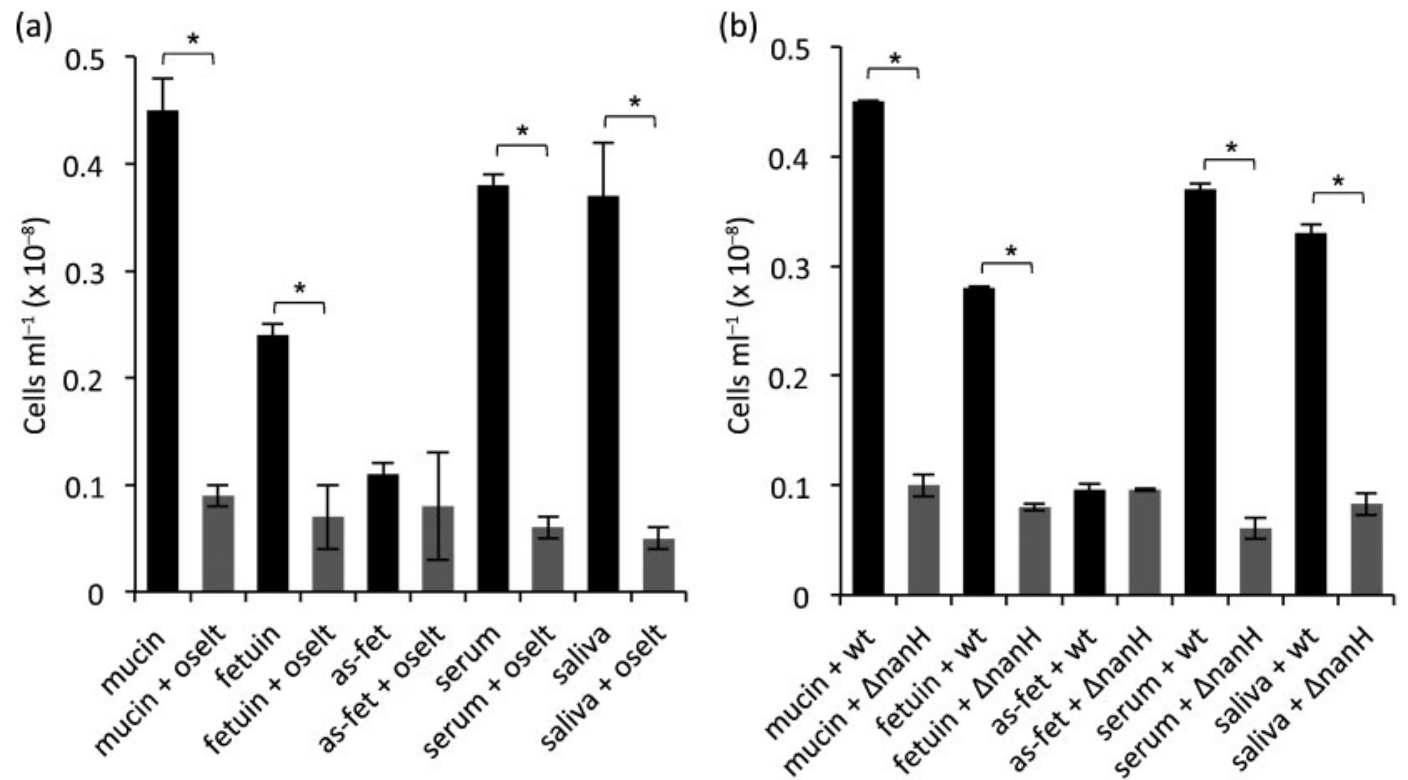

Fig. 2. Initial attachment of $T$. forsythia to glycoprotein-coated surfaces. (a) Adhesion to glycoproteins was assessed for the wild-type strain in the absence (black bars) or presence of oseltamivir (grey bars). Biofilms were set up in triplicate and wells were supplemented with $6 \mathrm{mM}$ sialic acid (sialic), or were coated with $6 \mathrm{mM}$ mucin, fetuin and asialofetuin (as-fet), $2 \mu \mathrm{g}$ human serum $\mathrm{ml}^{-1}$ (serum) or saliva, with and without the addition of $10 \mathrm{mM}$ oseltamivir to the TSB medium, as indicated at the time of inoculation. Glycoproteins were coated on the 96 -well plate overnight at $4{ }^{\circ} \mathrm{C}$ and washed before inoculation with $T$. forsythia to a final $\mathrm{OD}_{600}$ of 0.05 . Data are the mean number of cells with SDs after harvesting from wells after $3 \mathrm{~h}$. Differences between mean values in this experiment were evaluated by $t$ test, with $P<0.05$ being taken as the level of significance ( ${ }^{*}$ statistically significant). (b) Adhesion to glycoproteins was compared as above between the wild-type (wt) strain and $\Delta$ nan $H$ mutant strain of $T$. forsythia $(\Delta \mathrm{nanH})$. 


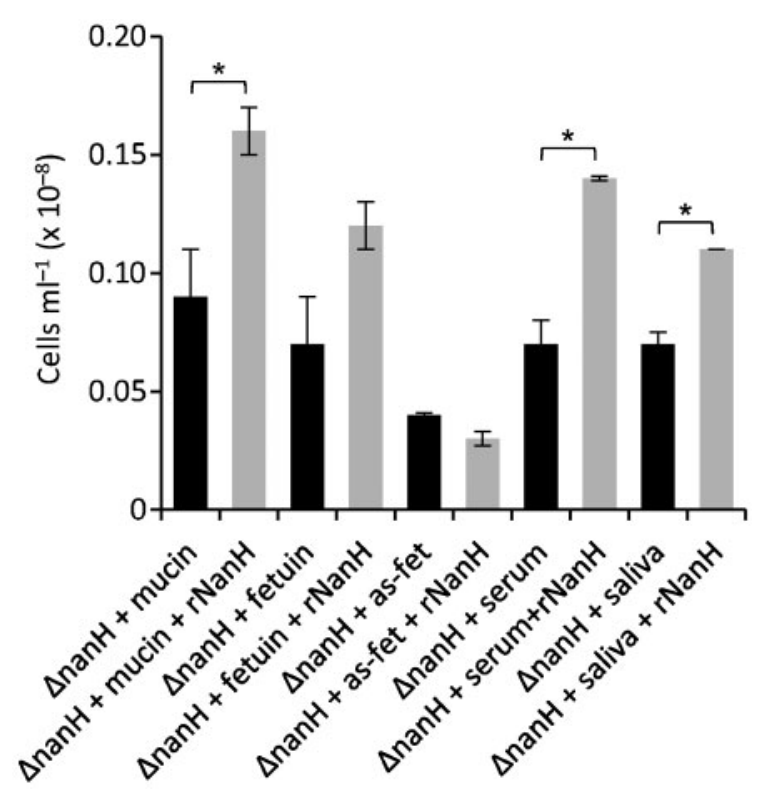

Fig. 3. Adhesion of the $\Delta n a n H$ mutant strain of $T$. forsythia to glycoprotein surfaces without (black bars) and with purified $\mathrm{NanH}$ $(\mathrm{rNanH})$ (grey bars). Biofilms were set up in triplicate and wells were supplemented with $6 \mathrm{mM}$ sialic acid (sialic) or $6 \mathrm{mM}$ sialyllactose (sia-lac) to the TSB medium, or were coated with $6 \mathrm{mM}$ mucin, fetuin and asialofetuin (as-fet), $2 \mu \mathrm{g}$ human serum $\mathrm{ml}^{-1}$ (serum) or saliva, as indicated at the time of inoculation. Glycoproteins were coated on the 96-well plate overnight at $4{ }^{\circ} \mathrm{C}$ and washed before inoculation with $T$. forsythia to a final $\mathrm{OD}_{600}$ of 0.05 . Data are the number of cells with SDs after harvesting from wells after $3 \mathrm{~h}$. Differences between mean values in this experiment were evaluated by $t$ test, with $P<0.05$ being taken as the level of significance ("statistically significant).

purified $\mathrm{rNanH}$ as described previously (Honma et al., 2011). After purification, the $\mathrm{rNanH}$ enzyme $(0.78 \mathrm{mg}$ $\mathrm{ml}^{-1}$ ) was incubated with $6 \mathrm{mM}$ fetuin and mucin for $3 \mathrm{~h}$ at $37{ }^{\circ} \mathrm{C}$. The release of sialic acid from these proteins was then assessed using the thiobarbiturate assay of Skoza \& Mohos (1976). Fig. 4 illustrates that in addition to cleavage of the 4-methylumbelliferyl substrate, we have now shown, to our knowledge for the first time, that rNanH can cleave sialic acid from the sialylated glycoprotein fetuin (Fig. 4, column 1) with the release of $30 \mathrm{nM}$ sialic acid $\mathrm{min}^{-1}(\mathrm{mg}$ protein $)^{-1}$. Furthermore, we showed that this is inhibitable by the sialidase inhibitor siastatin $\mathrm{B}$, indicating that this is a sialic acid-specific activity. When this enzyme was incubated with the same concentration of mucin $(6 \mathrm{mM}$ bovine submaxillary) (Fig. 4, column 4) we also observed a release of sialic acid. This result was somewhat surprising, since Thompson et al. (2009) reported that they could not detect release of sialic acid from mucin using rNanHexpressing lysates of $E$. coli. However, the concentration of mucin used in this assay $(6 \mathrm{mM})$ contained approximately 32 -fold more sialic acid than the equivalent concentration of fetuin. In contrast, if $\mathrm{rNanH}$ was incubated with

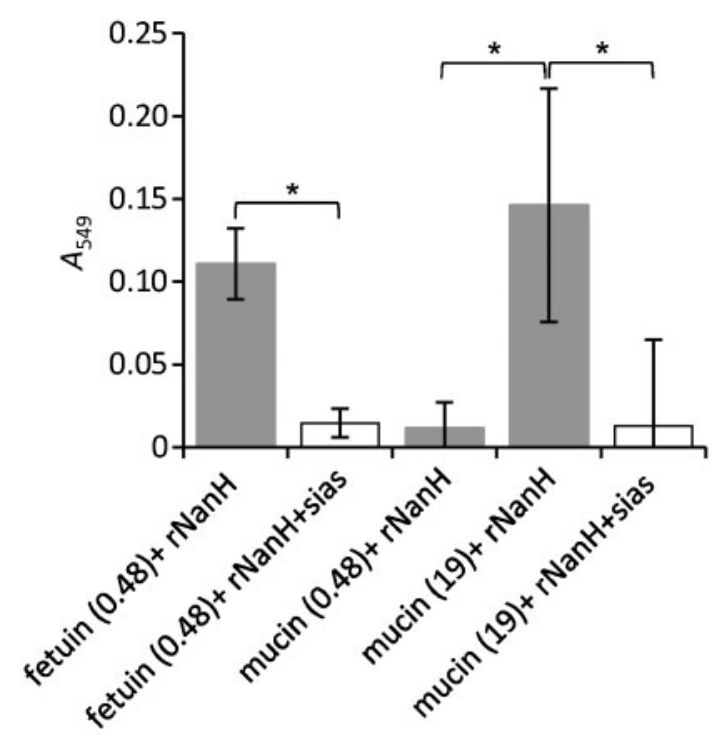

Fig. 4. Release of free sialic acid from glycoprotein by recombinant $\mathrm{NanH}(\mathrm{rNanH})$. Six millimolar fetuin $\left[0.48 \mu \mathrm{g}\right.$ sialic acid $\mathrm{ml}^{-1}$, fetuin (0.48)], $0.002 \mathrm{mM}$ mucin [0.48 $\mu \mathrm{g}$ sialic acid $\mathrm{ml}^{-1}$, mucin (0.48)] or $6 \mathrm{mM}$ mucin [19 $\mu \mathrm{g}$ sialic acid $\mathrm{ml}^{-1}$, mucin (19)] was incubated in the presence (white bars) and absence (grey bars) of $5 \mathrm{mM}$ of the sialidase inhibitor siastatin $\mathrm{B}(+$ sias $)$ at $37{ }^{\circ} \mathrm{C}$ for $3 \mathrm{~h} . A_{549}$ was measured after colour development (*statistically significant at $P<0.05)$.

$0.002 \mathrm{mM}$ mucin, which contained the equivalent amount of sialic acid $\left(0.48 \mu \mathrm{g} \mathrm{ml}^{-1}\right)$ as fetuin, there was almost no release of sialic acid (Fig. 4, column 3).

In order to further confirm our observation that $\mathrm{rNanH}$ can release sialic acid from physiologically relevant substrates, we probed the glycoprotein fetuin that had been pretreated with $\mathrm{rNanH}$ for the presence of the epitope for the sialic acid-specific biotinylated SNA lectin, which was detected with streptavidin-HRP. The SNA lectin binds preferentially to sialic acid linked to terminal galactose in $\alpha-2,6$ and to a lesser extent $\alpha-2,3$ linkages, both of which are contained in fetuin. Fig. 5 shows that in the absence of $\mathrm{rNanH}$ (lane 1), the fetuin band can clearly be seen at $68 \mathrm{kDa}$. However, after the treatment of fetuin with increasing concentrations of purified $\mathrm{rNanH}$, the lectinbinding site was removed and the fetuin-specific band disappeared from the blots (Fig. 5). These data support our findings using the thiobarbituric acid assay, and demonstrate for the first time to our knowledge that T. forsythia $\mathrm{NanH}$ can cleave both $\alpha-2,6-$ and $\alpha$-2,3-linked sialic acid directly from glycoproteins.

\section{DISCUSSION}

Our previous work identified that the fastidious anaerobe T. forsythia is able to utilize sialic acid as a growth factor to stimulate its biofilm growth, and that it possesses a novel 


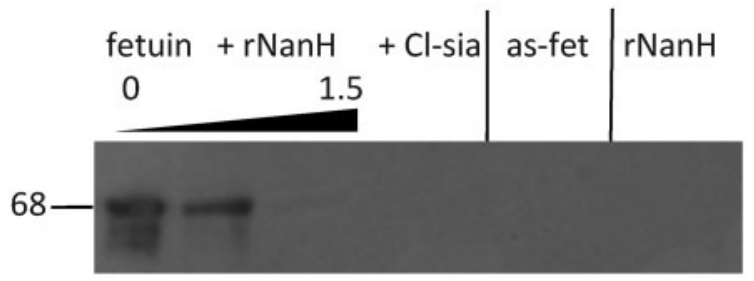

Fig. 5. Removal of lectin-binding moieties by $\mathrm{rNanH}$. Fetuin at $10 \mu \mathrm{g} \mathrm{ml}^{-1}$ was incubated with increasing concentrations of $\mathrm{rNanH}\left(0,0.78\right.$ and $\left.1.5 \mathrm{mg} \mathrm{m}^{-1}\right)$ for $3 \mathrm{~h}$ before running on an SDS-PAGE gel, blotting onto a nitrocellulose membrane, probing with $5 \mu \mathrm{g} \mathrm{ml}^{-1}$ biotinylated SNA lectin, and visualizing with streptavidin-HRP and luminescent substrate, before incubation with X-ray film. Clostridium tetani sialidase (Cl-sia) (50 U) was used as a positive control, with asialofetuin (as-fet) and $1.5 \mathrm{mg}$ $\mathrm{rNanH} \mathrm{ml} \mathrm{m}^{-1}$ as negative controls.

sialic uptake transport system (Roy et al., 2010). We have also shown that the sialidase activity of $T$. forsythia is important for sialic acid acquisition from the common glycosyl mimic sialyl-lactose (Roy et al., 2010). In other work we have established that the $\mathrm{NanH}$ sialidase is involved in adhesion to and invasion into gingival epithelial cells (Honma et al., 2011). We now report that the $\mathrm{NanH}$ sialidase is key to the initial formation and growth of biofilms on glycoprotein-coated surfaces, where the only source of sialic acid was conjugated to glycoproteins as $\alpha-2,3$ or 2,6 linkages to galactose or glucose. This is particularly pertinent given the environmental niche that T. forsythia inhabits in the oral cavity, where there is an abundance of sialic acid-containing glycoproteins that include fibronectin, integrins, gangliosides and mucins, both in secretions and contained on the surface of oral epithelial cells (Gabriel et al., 2005; Byres et al., 2008; Martin et al., 2005; Offner \& Troxler, 2000; Dabelsteen, 1996). The role of sialidases in the release of nutritionally available sialic acid is similar to the strategy of several human-dwelling bacteria in mucin-rich environments, such as the intestinal (Bacteroides fragilis and Vibrio cholerae) and respiratory tracts (S. pneumoniae) (Corfield, 1992).

Our data highlight roles for conjugated sialic acid both as a source of nutrition and as a receptor in the initial stages of biofilm formation on glycoprotein-coated surfaces, a situation which may mimic in vivo conditions in which inert and cellular surfaces are coated in a layer of mucus (Derrien et al., 2010). While binding of oral bacteria such as Streptococcus gordonii and Streptococcus sanguis to the sialic acid in mucin has been documented in the past (Demuth et al., 1990; Takahashi et al., 2002, 2004), we believe that this is the first indication that a Gram-negative periodontal pathogen also possesses such an activity. Our finding that both sialidase inhibitors and deletion of the sialidase gene affect adhesion to glycoproteins suggests that three separate mechanisms may be occurring simultaneously to achieve adhesion: (1) the sialidase itself acts as a lectin-like adhesin (Banerjee et al., 2010), perhaps via the 170 aa $\mathrm{N}$-terminal region; (2) the sialidase causes exposure of the underlying glucose and galactose residues to which the bacteria bind; (3) there is a two-stage mechanism in which sialic acid is key to the initial stages of adhesion but in which underlying sugars may be involved in more stable interactions. This is the case for some other pathogens, in which desialylated glycolipids can act as receptors for pathogenic bacteria, including $S$. pneumoniae and Pseudomonas aeruginosa, which bind to exposed GalNAc $\beta 1-4 \mathrm{Gal}$ residues when sialic acid is removed (Krivan et al., 1988). Taken overall and in light of our previous work showing the importance of sialidase and sialic acid in adhesion to host cells, it seems that in vivo desialylation of host molecules is crucial to T. forsythia. Recent evidence from the Fletcher laboratory has highlighted that this may also be the case for the other major periodontal pathogen $P$. gingivalis, in which mutations in a putative sialidase and two sialyl-endopeptidases affect adhesion to HeLa cells (Aruni et al., 2011); however, at present there is no evidence regarding their role in biofilm formation in $P$. gingivalis. Similarly, in the oral setting, the sialidase activity of several Streptococcus spp. can also result in exposure of galactose residues, enhancing the attachment of bacteria that possess galactosyl-binding adhesins, e.g Fusobacterium spp. (Falkler \& Burger, 1981) and Actinomyces viscosus (Ellen et al., 1980). It now seems possible that $T$. forsythia may also play such a role.

While the role of sialidase in $T$. forsythia adhesion and nutrition seems well established, like many members of the Bacteroidetes, $T$. forsythia possesses a plethora of glycosidase and sugar-focussed metabolic capabilities. For example $T$. forsythia possesses at least three putative betahexosaminidase-encoding genes (TF0036, TF2925 and TF0014), which may possess the ability to remove terminal galactose and glucose sugars from glycoproteins that are exposed after removal of sialic acid (Spiro \& Bhoyroo, 1974; Salyers et al., 1988). However, at present, the role of these enzymes in the biofilm lifestyle of $T$. forsythia is unknown.

The action of sialidase may also have nutritional consequences for other oral biofilm inhabitants; for example, $S$. oralis releases sialic acid from $\alpha_{1}$-acid glycoproteins (AGPs), which are then utilized by other bacteria (Byers et al., 1999). Similarly, after the removal of sialic acid from fetuin and mucin, subsequent removal of galactose and glucose can be used by bacteria as a carbon and nitrogen source (Leake \& Read, 1990). Indeed, this may be the case for $T$. forsythia, since subgingival plaque is made up of several species with the ability to use sialic acid and other sugars, while $T$. forsythia itself probably cannot.

In addition to our experiments highlighting adhesion to and biofilm growth upon glycoprotein-coated surfaces, we also showed by biochemical and lectin-binding assays that the $\mathrm{rNanH}$ enzyme releases $\alpha$-2,6-linked sialic acid from 
fetuin. This was expected, given the fact that $\mathrm{NanH}$ has been shown to preferentially cleave sialic acid from 2,6 sialyl-lactose in vitro. Our finding that sialic acid release from the $\alpha$-2,3- and $\alpha$-2,6-linked but largely 9-O-acetylated sialic acid contained in mucin was much less efficient is also in keeping with earlier findings, since this chemical group is known to inhibit bacterial sialidases (Thompson et al., 2009).

However, our data show that T. forsythia is able to grow as a biofilm on mucin-coated surfaces and indicate that it is thus able to remove the 9-O-acetyl group from the sialic acid contained on the surface of mucin (Fig. 4). This is probably due to the activity of a putative secreted 9-Oacetylesterase encoded by the TF0037 gene, and may play a crucial role in vivo in the mucin-rich oral environment that T. forsythia inhabits. In the gut bacterium E. coli, mutation of a recently identified sialate- $O$-acetyl esterase, nanS, results in abolition of its ability to use 9-O-acetylated sialic acid as a sole carbon and energy source. However, the NanS of E. coli, which does not possess a secreted sialidase, is a predicted periplasmic protein that probably acts to process Neu5,9Ac during sialic acid uptake (Steenbergen et al., 2009). In contrast, the putative NanS of T. forsythia is likely to be secreted into the extracellular milieu, where it could help with scavenging of sialic acid from host sources.

Overall, our findings indicate that sialoglycoproteins are important substrates for biofilm formation and attachment of $T$. forsythia to oral surfaces in vivo. In addition, this study raises the possibility that oseltamivir or siastatin B might be used as alternative drug therapies for reduction of dental plaque biofilms.

\section{ACKNOWLEDGEMENTS}

This work was funded by grants from the Royal Society, Dunhill Medical Trust and British Oral and Dental Research Trust to G.P.S., and by a University of Sheffield Oral Disease cluster studentship and Wellcome Trust Value in People Fellowship to S.R. The work in A. S.'s laboratory was funded by US Public Health Grant DE014749.

\section{REFERENCES}

Angata, T. \& Varki, A. (2002). Chemical diversity in the sialic acids and related $\alpha$-keto acids: an evolutionary perspective. Chem Rev 102, 439-470.

Aruni, W., Vanterpool, E., Osbourne, D., Roy, F., Muthiah, A., Dou, Y. \& Fletcher, H. M. (2011). Sialidase and sialoglycoproteases can modulate virulence in Porphyromonas gingivalis. Infect Immun 79, 2779-2791.

Banerjee, A., Van Sorge, N. M., Sheen, T. R., Uchiyama, S., Mitchell, T. J. \& Doran, K. S. (2010). Activation of brain endothelium by pneumococcal neuraminidase NanA promotes bacterial internalization. Cell Microbiol 12, 1576-1588.

Bradshaw, D. J., Homer, K. A., Marsh, P. D. \& Beighton, D. (1994). Metabolic cooperation in oral microbial communities during growth on mucin. Microbiology 140, 3407-3412.
Bradshaw, D. J., Marsh, P. D., Watson, G. K. \& Allison, C. (1998). Role of Fusobacterium nucleatum and coaggregation in anaerobe survival in planktonic and biofilm oral microbial communities during aeration. Infect Immun 66, 4729-4732.

Byers, H. L., Tarelli, E., Homer, K. A. \& Beighton, D. (1999). Sequential deglycosylation and utilization of the $N$-linked, complextype glycans of human alphal-acid glycoprotein mediates growth of Streptococcus oralis. Glycobiology 9, 469-479.

Byres, E., Paton, A. W., Paton, J. C., Löfling, J. C., Smith, D. F., Wilce, M. C., Talbot, U. M., Chong, D. C., Yu, H. \& other authors (2008). Incorporation of a non-human glycan mediates human susceptibility to a bacterial toxin. Nature 456, 648-652.

Cointe, D., Leroy, Y. \& Chirat, F. (1998). Determination of the sialylation level and of the ratio $\alpha-(2 \rightarrow 3) / \alpha-(2 \rightarrow 6)$ sialyl linkages of $\mathrm{N}$-glycans by methylation and GC/MS analysis. Carbohydr Res 311, 51-59.

Corfield, T. (1992). Bacterial sialidases-roles in pathogenicity and nutrition. Glycobiology 2, 509-521.

Dabelsteen, E. (1996). Cell surface carbohydrates as prognostic markers in human carcinomas. J Pathol 179, 358-369.

Demuth, D. R., Golub, E. E. \& Malamud, D. (1990). Streptococcal-host interactions. Structural and functional analysis of a Streptococcus sanguis receptor for a human salivary glycoprotein. J Biol Chem $\mathbf{2 6 5}$, $7120-7126$.

Derrien, M., van Passel, M. W., van de Bovenkamp, J. H., Schipper, R. G., de Vos, W. M. \& Dekker, J. (2010). Mucin-bacterial interactions in the human oral cavity and digestive tract. Gut Microbes 1, 254-268. El-Kheshen, M. (2009). Plaque plague. Dentist December 2008, 4850 .

Ellen, R. P., Fillery, E. D., Chan, K. H. \& Grove, D. A. (1980). Sialidaseenhanced lectin-like mechanism for Actinomyces viscosus and Actinomyces naeslundii hemagglutination. Infect Immun 27, 335-343.

Falkler, W. A., Jr \& Burger, B. W. (1981). Microbial surface interactions: reduction of the haemagglutination activity of the oral bacterium Fusobacterium nucleatum by absorption with Streptococcus and Bacteroides. Arch Oral Biol 26, 1015-1025.

Gabriel, M. O., Grünheid, T. \& Zentner, A. (2005). Glycosylation pattern and cell attachment-inhibiting property of human salivary mucins. J Periodontol 76, 1175-1181.

Honma, K., Mishima, E. \& Sharma, A. (2011). Role of Tannerella forsythia NanH sialidase in epithelial cell attachment. Infect Immun 79, 393-401.

King, S. J., Hippe, K. R. \& Weiser, J. N. (2006). Deglycosylation of human glycoconjugates by the sequential activities of exoglycosidases expressed by Streptococcus pneumoniae. Mol Microbiol 59, 961-974.

Kolenbrander, P. E. \& Palmer, R. J. (2004). Human oral bacterial biofilms. In: Microbial Biofilms, pp. 85-117. Edited by M. A. Ghannoum \& G. A. O'Toole. Washington, DC: American Society for Microbiology.

Krivan, H. C., Roberts, D. D. \& Ginsburg, V. (1988). Many pulmonary pathogenic bacteria bind specifically to the carbohydrate sequence GalNAc beta $1-4 \mathrm{Gal}$ found in some glycolipids. Proc Natl Acad Sci U S A 85, 6157-6161.

Kuroiwa, A., Hisatsune, A., Isohama, Y. \& Katsuki, H. (2009). Bacterial neuraminidase increases IL-8 production in lung epithelial cells via NF- $\kappa$ B-dependent pathway. Biochem Biophys Res Commun 379, 754-759.

Leake, J. R. \& Read, D. J. (1990). Chitin as a nitrogen source for micorrhizal fungi. Mycol Res 94, 993-995.

Martin, M. J., Rayner, J. C., Gagneux, P., Barnwell, J. W. \& Varki, A. (2005). Evolution of human-chimpanzee differences in malaria 
susceptibility: relationship to human genetic loss of $N$-glycolylneuraminic acid. Proc Natl Acad Sci U S A 102, 12819-12824.

Mizan, S., Henk, A., Stallings, A., Maier, M. \& Lee, M. D. (2000). Cloning and characterization of sialidases with $2-6^{\prime}$ and $2-3^{\prime}$ sialyl lactose specificity from Pasteurella multocida. J Bacteriol 182, 68746883.

Offner, G. D. \& Troxler, R. F. (2000). Heterogeneity of highmolecular-weight human salivary mucins. Adv Dent Res 14, 69-75.

Pham, T. K., Roy, S., Noirel, J., Douglas, I., Wright, P. C. \& Stafford, G. P. (2010). A quantitative proteomic analysis of biofilm adaptation by the periodontal pathogen Tannerella forsythia. Proteomics 10, 3130-3141.

Pigman, W. \& Gottschalk, A. (1966). Submaxillary gland glycoproteins. In Glycoproteins. Their Composition, Structure, and Function, pp. 434-445. Amsterdam, The Netherlands: Elsevier.

Reinholdt, J., Tomana, M., Mortensen, S. B. \& Kilian, M. (1990). Molecular aspects of immunoglobulin A1 degradation by oral streptococci. Infect Immun 58, 1186-1194.

Roy, S., Douglas, C. W. \& Stafford, G. P. (2010). A novel sialic acid utilization and uptake system in the periodontal pathogen Tannerella forsythia. J Bacteriol 192, 2285-2293.

Salyers, A. A., Pajeau, M. \& McCarthy, R. E. (1988). Importance of mucopolysaccharides as substrates for Bacteroides thetaiotaomicron growing in intestinal tracts of exgermfree mice. Appl Environ Microbiol 54, 1970-1976.

Scannapieco, F. A. (1994). Saliva-bacterium interactions in oral microbial ecology. Crit Rev Oral Biol Med 5, 203-248.

Severi, E., Hood, D. W. \& Thomas, G. H. (2007). Sialic acid utilization by bacterial pathogens. Microbiology 153, 2817-2822.

Skoza, L. \& Mohos, S. (1976). Stable thiobarbituric acid chromophore with dimethyl sulphoxide. Application to sialic acid assay in analytical de-O-acetylation. Biochem J 159, 457-462.
Socransky, S. S., Haffajee, A. D., Cugini, M. A., Smith, C. \& Kent, R. L., Jr (1998). Microbial complexes in subgingival plaque. J Clin Periodontol 25, 134-144.

Soong, G., Muir, A., Gomez, M. I., Waks, J., Reddy, B., Planet, P., Singh, P. K., Kaneko, Y., Wolfgang, M. C. \& other authors (2006). Bacterial neuraminidase facilitates mucosal infection by participating in biofilm production. J Clin Invest 116, 2297-2305.

Spiro, R. G. \& Bhoyroo, V. D. (1974). Structure of the O-glycosidically linked carbohydrate units of fetuin. J Biol Chem 249, 5704-5717.

Steenbergen, S. M., Jirik, J. L. \& Vimr, E. R. (2009). YjhS (NanS) is required for Escherichia coli to grow on 9-O-acetylated $\mathrm{N}$-acetylneuraminic acid. J Bacteriol 191, 7134-7139.

Takahashi, Y., Konishi, K., Cisar, J. O. \& Yoshikawa, M. (2002). Identification and characterization of $h s a$, the gene encoding the sialic acid-binding adhesin of Streptococcus gordonii DL1. Infect Immun 70, 1209-1218.

Takahashi, Y., Yajima, A., Cisar, J. O. \& Konishi, K. (2004). Functional analysis of the Streptococcus gordonii DL1 sialic acid-binding adhesin and its essential role in bacterial binding to platelets. Infect Immun 72, 3876-3882.

Tanner, A. C. \& Izard, J. (2006). Tannerella forsythia, a periodontal pathogen entering the genomic era. Periodontol 2000 42, 88-113.

Thompson, H., Homer, K. A., Rao, S., Booth, V. \& Hosie, A. H. (2009). An orthologue of Bacteroides fragilis $\mathrm{NanH}$ is the principal sialidase in Tannerella forsythia. J Bacteriol 191, 3623-3628.

Tong, H. H., Blue, L. E., James, M. A. \& DeMaria, T. F. (2000). Evaluation of the virulence of a Streptococcus pneumoniae neuraminidase-deficient mutant in nasopharyngeal colonization and development of otitis media in the chinchilla model. Infect Immun 68, 921-924.

Varki, A. (1997). Sialic acids as ligands in recognition phenomena. FASEB J 11, 248-255.

Edited by: W. Crielaard 